\title{
Zum Bernsteinschen Satz für Quasiminimalflächen
}

\author{
E. HOY
}

A generalization of Bernstein's theorem is proved for (parametric) surfaces with a quasiconformal Gauss map.

Key words: Berustein's theorem, parametric quasiminimal surface

AMS subject classification: $53 \wedge 10,30 \subset 60$

1. Einleitung. In der vorliegenden Arbeit soll der folgende Satz bewiesen werden.

Satz: Es sej $S \subset \mathbb{R}^{3}$ eine einfach zusammenhängende, reguläre (also orientierbare) und vollständige Fläche mit einer dreimal stetig differenzierbaren Parameterdarstellung $x=\left(x_{1}(u, v), x_{2}(u, v), x_{3}(u, v)\right)$, wobei die Gaußsche Krümmung $K$ von $S$ stets nichtpositiv und die GauBsche Abbildung von $S$ noch $Q$-quasikonform mit einem $Q \in[1,3)$ sei. Wenn das Bild bei der Gaußschen Abbildung vollständig in einer Hemisphäre liegt, so kann $S$ nur eine Ebene sein.

Flächen mit nichtpositiver Gaußscher Krümmung und quasikonformer Gaußscher Abbildung werden nach [13] Quasiminimalflächen genannt in Verallgemeinerung des Begriffes Minimalfläche, die ihrerseits eine konforme Gaußsche Abbildung besitzen. Man kann ohne Beschränkung der Allgemeinheit annehmen, daß es sich in Satz 1 um die untere (suidliche) Hemisphäre handelt. Satz 1 verallgemeinert somit den Bernsteinschen Satz fur Minimalflächen, nämlich, daß jede Minimalfläche in expliziter (nichtparametrischer) Form

$$
x_{3}=f\left(x_{1}, x_{2}\right) \text { für alle } x_{1}, x_{2} \in \mathbf{R}
$$

eine Ebene sein muß. Dazu wird auf [3] oder auch auf [2: Chapter III], [10], [11: § 751] und [12] verwiesen. Andererseits hängt Satz 1 eng mit einem Ergebnis von L. Simon (siehe [14] oder [7:Chapter 15.6]) zusammen, der den Bernsteinschen Satz auch für Quasiminimalflächen verallgemeinert hat. Allerdings wird im Gegensatz zum Satz 1 die Darstellung (1) benutzt, jedoch keine weitere Einschränkung an $Q \geq 1$ benötigt, wie es im Sat $z 1$ der Fall ist. Verknüpft man die Gaußsche Abbildung mit der stereographischen Projektion in die komplexe $g$-Ebene, so läßt sich Satz 1 noch etwas allge meiner formulieren.

Satz 2: Es sei $S \subset \mathbb{R}^{3}$ eine einfach zusammenhängende, reguläre, orientierbare und vollständige Fläche mit einer dreimal stetig differenzierbaren Parameterdarstellung, wobei die GauBsche Kriummung von $S$ stets nichtpositiv und die GauBsche Abbildung von $S$ noch $Q$-quasikonform mit einem $Q \in[1,8)$ sei. Wenn die stereographische Projektion des sphärischen Bildes von $S$ in der Krejsscheibe $\left\{w:|w|^{2} \leq C\right\}$ liegt und die Ungleichungen

$$
O C<4\left(1-(1+C)^{-2}\right)
$$

sowie

$0<C<3,828424 \ldots$

gelten, so muß $S$ eine Ebene sein. 
2. Hilfsmittel. In diesem Abschnitt werden für die Beweise der Sätze 1 und 2 einige Hilfsmittel zusammengestellt. Auf $S$ werden im folgenden geodätische Polarkoordinaten $r, \vartheta$ mit $0 \leq r<\infty$ und $o \leq \vartheta \leq 2 \pi$ eingeführt. Wegen $K \leq 0$ kann man diese Koordinaten nach der Wahl eines festen Punktes $P_{0}$ mit $r=0$ auf $S$ sogar global einfuihren (siehe dazu [4] oder auch [6: Kap.VI, §5]). Auf Grund der Vollständigkeit von $S$ wird $S$ umkehrbar eindeutig auf die Ebene mit den (gewöhnlichen) Polarkoordinaten $r, \vartheta$ abgebildet. Die Koordinatenfunktionen $x_{j}=x_{j}(r, \vartheta)$ mit $j=1,2,3$ genügen bekanntlich den Gleichungen

$$
\sum_{j=1}^{3} x_{j r}^{2}=1, \quad \sum_{j=1}^{3} x_{j r} x_{j \theta}=0, \quad \sum_{j=1}^{3} x_{j \theta}^{2}=G^{2}=G^{2}(r, \vartheta)
$$

mit einer nichtnegativen Funktion $G=G(r, \vartheta)$, die ihrerseits die Eigenschaften

$$
G(0,9)=0, G_{r}(0,9)=1
$$

hat, wie man aus [4:S. 302], [5:Kap.4.6] und [6:Kap.VI, §4] entnehmen kann. Die Gaußsche Krümmung $K$ berechnet sich aus (siehe ebenda)

$$
K=-G_{r r} / G
$$

so daß damit für die Funktion

$$
L(r)=\int_{0}^{2 \pi} G(r, \vartheta) d \vartheta
$$

wegen $K \leq 0$ noch $L^{\prime \prime}(r) \geq 0$ für alle $r \in[0, \infty)$ gilt.

Es sei nun $g=g_{1}+\mathrm{i} g_{2}=g_{1}(r, \vartheta)+i g_{2}(r, \vartheta)$ die Funktion, die durch Verknüpung der Abbildung der Ebene mit den Polarkoordinaten $r, \vartheta$ auf $S$ mit der Gaußschen Abbildung und der stereographischen Projektion in die $g$-Ebene entsteht, wobei $g_{1}$ und $g_{2}$ reellwertig seien. Dann ist die $Q$-Quasikonformität der Gaußschen Abbildung äquivalent zur Ungleichung

$$
g_{1 r}^{2}+g_{2 r}^{2}+\frac{g_{1 \theta}^{2}+g_{2 \theta}^{2}}{G^{2}} \leq\left(Q+\frac{1}{Q}\right) \frac{\left|g_{1 r} g_{2 \theta}-g_{2 r} g_{1 \theta}\right|}{G} \quad \forall r>0, \vartheta \in[0,2 \pi],
$$

wie man mittels (4) zeigen kann. Desweiteren gilt für $T=g_{2 r} g_{1 \vartheta}-g_{1 r} g_{2 \theta}$ wegen (6) noch

$$
\left(1+|g|^{2}\right)^{2} G_{r r} / 4=T,
$$

so daß aus $K \leq 0$ sogar $T \geq 0$ folgt.

3. Beweis der Sütze 1 und 2. Da Satz 1 ein Spezialfall des Satzes 2 für $C=1$ ist, genügt es, Satz 2 zu beweisen. Wie in [10:Formel(10)] erhält man mit Hilfe des Stokesschen Integralsatzes bei Benutzung von $T=g_{2 r} g_{1 \vartheta}-g_{1 r} g_{2 \vartheta}$ die Identität

$$
\int_{0}^{R} \int_{0}^{2 \pi} \varphi^{2}(r) T d r d \vartheta=\int_{0}^{R} \int_{0}^{2 \pi} \varphi(r) \varphi^{\prime}(r)\left(g_{1} g_{2 \vartheta}-g_{2} g_{1 \vartheta}\right) d r d \vartheta
$$

für jede in $[0, R]$ stetig differenzierbare reellwertige Funktion $\varphi=\varphi(r)$ mit $\varphi(R)=0$. Die rechte Seite von (10) kann mit Hilfe der Ungleichung

$$
\left|\varphi(r) \varphi^{\prime}(r)\right|\left|g_{1} g_{2 \vartheta}-g_{2} g_{1}\right| \leq \beta Q G \varphi^{\prime 2}(r)+\varphi^{2}(r)|g|^{2} \mid g_{\vartheta}{ }^{2} / 4 \beta Q G
$$

nach oben abgeschätzt werden, wobei die Konstante $\beta>1 / 8$ sein soll. Nutzt man noch die sich aus (8) ergebende Ungleichung $\left|g_{\theta}\right|^{2} \leq Q G T$, so folgt schließlich

$$
\int_{0}^{R} \int_{0}^{2 \pi} \varphi^{2}(r) T\left(1-|g|^{2} / 4 \beta\right) d r d \vartheta \leq \beta Q \int_{0}^{R} \int_{0}^{2 \pi} \varphi^{\prime 2}(r) G d r d \vartheta
$$

Der Integrand auf der linken Seite kann mittels $(9)$ in $\varphi^{2}(r) G_{r r}\left(1+|g|^{2}\right)^{2}\left(1-|g|^{2 / 4 \beta}\right) / 4$ umgeformt werden. Man definiert nun eine Zahl $C>0$ als Lösung von $(1+C)^{2}(1-C / 4 \beta)=1$. Eine solche existiert für alle $\beta>1 / 8$ und ist zudem eindeutig bestimmt. Des weiteren gilt 
dann für alle komplexen $g$ mit $0 \leq|g|^{2} \leq C$ sogar $\left(1+|g|^{2}\right)^{2}\left(1-|g|^{2} / 4 \beta\right) \geq 1$. Auf diese Weise folgt aus (11) schließlich $\int_{0}^{R} \int_{0}^{2 \pi} \varphi^{2}(r) G_{r r} d r d \vartheta \leq 4 \beta Q \int_{0}^{R} \int_{0}^{2 \pi} \varphi^{\prime 2}(r) G d r d 9$ bzw.wegen (7)

$$
\int_{0}^{R} \varphi^{2}(r) L^{\prime \prime}(r) d r \leq 4 \beta Q \int_{0}^{R} \varphi^{\prime 2}(r) L(r) d r .
$$

Das ist eine notwendige Bedingung für die $Q$-Quasikonformität derGaußschen Abbildung Im folgenden wird die noch freie Größe $\beta>1 / 8$ so gewählt, da $B Q<1$ gilt. Dann ergibt sich $Q<8$, und aus $(1+C)^{2}(1-C / 4 \beta)=1$ folgt die Ungleichung (2). Die obere Schranke von $C$ in (3) erhält man durch das Lösen von $(1+C)^{2}(1-C / 4)=1$, d.h. im Grenzfall $\beta=1$.

Nun muß nur noch gezeigt werden, daß die Guiltigkeit von (12) im Fall $\beta Q<1$ auf $L^{\prime \prime}(r)$ $\equiv 0$ führt. Die Ungleichung (12) gilt nach entsprechenden Approximationsbetrachtungen auch fuir die Funktion $\varphi$ mit $\varphi(r)=\int_{\rho}^{R} L^{-1 / 2}(t) d t=$ const für $0 \leq r \leq \rho$ und $\varphi(r)=\int_{r}^{R} L^{-1 / 2}(t) d t$ für $\rho<r \leq R$, wobei $\rho \in(0, R)$ beliebig gewählt wird. Die linke Seite von (12) ergibt nach zweimaliger Anwendung der partiellen Integration wie in [10:Beweis von Lemma1] die Größe

$$
-2 \pi\left(\int_{\rho}^{R} L^{-1 / 2}(r) d r\right)^{2}-4 L^{1 / 2}(\rho) \int_{\rho}^{R} L^{-1 / 2}(r) d r+4(R-\rho) .
$$

Andererseits gilt $\int_{\rho}^{R} \varphi^{\prime 2}(r) L(r) d r=\int_{\rho}^{R} d r=R-\rho$, d.h., aus (12) folgt mit $I=\int_{\rho}^{R} L^{-1 / 2}(r) d r$ noch $-2 \pi I^{2}-4 L^{1 / 2}(\rho) I+(4-4 \beta Q)(R-\rho) \leq 0$. Das ist wegen $I>0$ und $\beta Q<1$ äquivalent $z u$

$$
I \geq-L^{1 / 2}(\rho) / \pi+\left(L(\rho) / \pi^{2}+(2-2 \beta Q)(R-\rho) / \pi\right)^{1 / 2} \text {. }
$$

Da die Funktion $L(r) / r$ wegen $L(0)=0$ und $L^{\prime \prime}(r) \geq 0$ in $[0, R]$ monoton nichtfallend ist, gilt $I s \int_{\rho}^{R} \rho^{1 / 2} L^{-1 / 2}(\rho) r^{-1 / 2} d r=2(\rho / L(\rho))^{1 / 2}\left(R^{1 / 2}-\rho^{1 / 2}\right)$. Zusammen mit (13) ergibt dies

$$
2(\rho / L(\rho))^{1 / 2}\left(R^{1 / 2}-\rho^{1 / 2}\right) \geq-L^{1 / 2}(\rho) / \pi+\left(L(\rho) / \pi^{2}+(2-2 \beta Q)(R-\rho) / \pi\right)^{1 / 2} \text {. }
$$

Die Division durch́ $R^{1 / 2}$ und der Grenzprozeß $R \rightarrow \infty$ liefern dann $\rho / L(\rho) \geq(1-\beta Q) / 2 \pi$, d.h., die Funktion $L(r) / r$ ist fuir alle $r \geq 0$ nach oben beschränkt. Daraus folgt wie in [10: Beweis von Lemma 1] durch das Einsetzen einer stuickweise linearen Funktion in $\ln r$ für $\varphi=\varphi(r)$ schließlich $L^{\prime \prime}(r) \equiv 0$.Wegen (7) und (9) verschwindet daher die Funktionaldeterminante der Abbildung $g=g_{1}(r, \vartheta)+i g_{2}(r, \vartheta)$ überall. Somit ergibt sich $g \equiv$ const, also die Behauptung des Satzes

4. Bomerkungen. 1. Der hier dargestellte Beweisgang hat wesentlich die Vorgehensweise in [10] benutzt. Jedoch ist das Ergebnis in [10] nur ein Spezialfall des Satzes von L. Simon (siehe [14] oder [7: Chapter 15.6]). Auch in der vorliegenden Arbeit sind nur Teilresultate bewiesen worden. wie besonders durch die Grenzfälle $C \rightarrow \infty$ (d.h. $Q \rightarrow 8-0$ ) und $C \rightarrow$ $3,828424 \ldots-O(d . h . Q \rightarrow 1+0)$ deutlich wird. Im zweiten Fall ist ein Vergleich mit [12] angebracht, wo Satz 2 für $Q=1$ und für beliebig großes (aber endliches) $C$ bewiesen wird.

2. Es soll hier auch bemerkt werden, daß der Beweis von Satz 2 nicht ohne weiteres furr wesentlich gröBere $O$ und $C$ als $8 \mathrm{bzw}, 3,82 \ldots$ durchfuhrbar ist. Beispielsweise gilt für die Funktion $L, L(r)=2 \pi\left(r+r^{n}\right)$ mit $n>2$, die Ungleichung

$$
\int_{0}^{R} L^{\prime \prime}(r) \varphi^{2}(r) d r \leq 4 \pi /(n-1) \int_{0}^{R} L(r) \varphi^{-2}(r) d r .
$$

wobei $\varphi=\varphi(r)$ eine beliebige in $[0, R]$ stetig differenzierbare reellwertige Funktion mit $\varphi(R)=0$ ist und $R>0$ beliebig gewählt werden kann. Vergleicht man (14) mit (12), so ist ersichtlich, daß die jeweils auf der rechten Seite vor dem Integral stehenden Konstanten von verschiedenen Richtungen der Zahl 4 uneingeschränkt nahe kommen können. Es ist allerdings offen, ob es eine Fläche $S$ mit einer solchen Funktion $L$ gibt und ob diese Fläche den Voraussetzungen von Satz 2 (ohne die oberen Schranken für $Q$ und $C$ ) genügt. 
3. Die hier beliandelte Problematik hängt eng mit dem Typenproblem für die Fläche $S$ zusammen. S ist nämlich wegen $K \leq 0$ entweder vom parabolischen oder hyperbolischen Typ. Im ersten Fall gilt Satz 2 sogar für alle $Q \geq 1$ und $C>0$. Es ist also nur zu zeigen, daß $S$ vom parabolischen Typ ist. Dafür erweist sich nach [1] die Divergenz von $\int_{1}^{\infty} L^{-1}(r) d r$ als hinreichend. Mit der hier benutzten Methode laßßt sich sogar der parabolische Typ von $S$ nachweisen, wenn nur $\int_{1}^{\infty} L^{-\alpha}(r) d r$ für alle $\alpha<1$ divergiert.

4. In [9] findet man einen anderen Weg, um aus (12) das Verschwinden von $L "(r)$ zu erhalten. Dort wird gezeigt, daB zu jeder $Z a h l c<4$ eine $Z a h 1 R>0$ und eine in $[0, R]$ stetig differenzierbare reelwertige Funktion $\psi=\psi(r)$ mit $\psi(R)=0$ existiert, so daß $\int_{0}^{R} \Psi^{2}(r) L^{\prime \prime}(r) d r$ $>c \int_{0}^{R} \Psi^{-2}(r) L(r) d r$ gilt, wenn die Fläche $S$ regulär, dreimal stetig differenzierbar und vollständig sowie die Gaußsche Krimmung von $S$ stets nichtpositiv und nicht identisch Null ist.

5. Die Voraussetzungen "orientierbar" und "einfach zusammenhängend" können in den Sätzen 1 und 2 nur durch "zusammenhangend" ersetzt werden, ohne daß sich in diesen Sätzen noch etwas ändern würde. Diese gängige Uberlegung wird beispielsweise in [11: $\$ 53$ und $\$ 147]$ und in $[12,13]$ ebenfalls verwendet.

6. Da die notwendige Bedingung für die Quasikonformität von $B$ in (12) mit Hilfe von Integralen gegeben ist, liegt es auch nahe, nach Abschwächungen von (8) zu suchen, ohne daß (12) verletzt wird. Ein solcher Gedanke hat in [8] Anwendung gefunden.

\section{LITERATUR}

[1] Ahlfors, L.V.: Sur le type dune surface de Riemann. C.R. Acad. Sci. Paris 201 (1935), $30-32$.

[2] BARBOSA, J.L., and A.G.COLARES: Minimal Surfaces in $\mathbb{R}^{3}$. Lect. Notes Math. 1195 (1980).

[3] BERNSTEIN, S.: Sur un théorème de géométrie et son applieation aux équations aux dérivées partielles du type elliptique. Comm. Soc. Math. Kharkov $15(1915-17), 38-45$.

[4] BIEBERBACH, L.: Uber Tschebyschefsche Netze auf Flächen negativer Krummung, sowie auf einigen weiteren Flachenarten. Sitzungsber. PreuB. Akad. Wiss. 23 (1926), 294 - 321.

[5] CARMO, M.P.: Differentialgoometrie von Kurven und Flächen. Braunschweig - Wiesbaden: Viewer 1983

[6] DUSCHEK, A., und W.MAYER: Lehrbuch der Differentialgeometrie I. Leipzig-Berlin: Teubner - Verlag 1930.

[7] GILBARG, D., und N.S.TRUDINGER: Elliptic Partial Differential Equations of Second Order. Berlin - Heidelberg - New York: Springer - Verlag 1977.

[8] HoY, E.: Cber eine Enwoiterung des Liouvilleschen Satzes. Z. Anal. Anw. 10 (1991)

[9] K^WAI, S.: Operator $\triangle$ - aK on surfaces. Hokkaido Math. J. 17 (1988), 147 - 150.

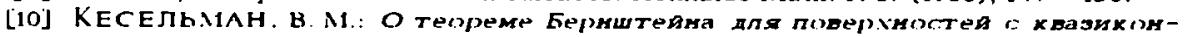
оррмнам гаустовым отображением. Мат. заметки 35 (1981). 445 - 453.

[11] NITSCHE, J.C.C.: Vorlesungen iber Minimalflächen. Berlin - Heidelberg - New York: Springer - Verlag 1975.

[12] OSSERMAN, R.: Proof of a conjecture of Nirenberg. Comm. Pure Appl.Math.12 (1959), $229-232$.

[13] OSSERM N, R.: On complete minimal surfaces. Arch. Rat. Mech. Anal. 13 (1963), 392 - 404.

[14] SIMON, L.: A Hölder estimate for quasiconformal maps berween surfaces in Euclidcan space. Acta math. 139 (1977), $19-51$.

Received 09.02.1990

Dr. Erich Hoy

Sektion Mathematik der Martin - Luther-Universität

Postfach

D (Ost) - 4010 Halle 